\title{
Review
}

\section{Longitudinal Neuroimaging Biomarkers in Huntington's Disease}

\author{
Elin M. Rees*, Rachael I. Scahill and Nicola Z. Hobbs \\ UCL Institute of Neurology, University College London, Queen Square, London, UK
}

\begin{abstract}
Identifying markers able to characterise the progression of Huntington's Disease (HD) is of great importance to the HD research community, as such markers may provide valuable outcome measures in future clinical trials. Neuroimaging measures are obvious candidates because of their clear relevance to the neuropathology of the disease. Many also show improved precision and sensitivity compared with standard functional scales. This review summarizes findings from the wealth of longitudinal imaging studies in the literature, focusing on the most widely available imaging modalities: structural MRI (volumetric and diffusion imaging), functional MRI and PET. We discuss the longitudinal sensitivity, reproducibility and feasibility of each imaging modality for use in clinical trials.
\end{abstract}

Keywords: Huntington's disease, longitudinal studies, biomarkers, magnetic resonance imaging, diffusion mri, functional mri, positron emission tomography

\section{INTRODUCTION}

Huntington's Disease (HD) is a genetic neurodegenerative disorder that affects approximately 6-7 per 100,000 people in the UK, although recent reports suggest the incidence could be higher [1]. It is caused by an expanded CAG triplet repeat in the Huntingtin gene [2] which results in progressive brain atrophy, most prominent in the striatum but also evident in widespread grey-matter (GM) and white-matter (WM) regions. CAG repeats of $40+$ show full penetrance $(100 \%$ of individuals are destined to develop HD) whereas incomplete penetrance is observed in the range of 35 to 39 repeats. HD manifests typically between 35 and 50 years of age with a triad of symptoms: motor, cognitive and psychiatric. Clinical diagnosis is based on the unequivocal presence of otherwise unexplained extrapyramidal movement disorder (e.g. chorea, bradykinesia, dystonia, rigidity) and

${ }^{*}$ Correspondence to: Elin Rees, UCL Institute of Neurology, University College London, c/o Box 104, Queen Square, London, WC1N 3BG, UK. Tel.: +440207 242 0038; Fax: +440207 611 0129; E-mail: elin.rees@ucl.ac.uk. death typically occurs between $15-20$ years post-onset. Prior to diagnosis in mutant gene-positive individuals (referred to here as preHD) there is a long period of preclinical decline in which signs and subtle symptoms of the disorder can be detected. Unlike many other neurodegenerative diseases where diagnosis is uncertain, the availability of a definite diagnostic test for HD allows research to be performed in preHD individuals.

HD is currently incurable, with only symptomatic treatments available. In recent years new therapies have undergone development and testing with the aim of slowing or attenuating disease progression [3], some of which have been successful in animal models of HD. With the commencement of disease-modifying clinical trials in humans, comes the need for reliable and sensitive biomarkers of disease progression which may prove useful as outcome measures. The Biomarkers Definitions Working Group defines a biomarker as, ' $a$ characteristic that is objectively measured and evaluated as an indicator of normal biological processes, pathogenic processes, or pharmacologic responses to a therapeutic intervention' [4]. Characteristics of an 
ideal biomarker include quantification which is reliable, reproducible across sites, minimally invasive and widely available. The biomarker should show low variability in the normal population and change linearly with disease progression, ideally over short time intervals. Finally, the biomarker should respond predictably to an intervention which modifies the disease.

Currently, standard clinical scales of symptom severity and tests of cognition are available as primary outcome measures. These include the: Unified HD Rating Scale (UHDRS [2]), Quantified Neurological Exam (QNE [5]) and neuropsychological testing (sensitive in clinical HD [6] but not preHD [7]). Unfortunately, high inter-rater variability [8], floor and ceiling effects, low sensitivity to longitudinal change and inability to easily discriminate between disease modification and symptomatic benefit are all limitations of clinical measures. Such limitations mean that well-powered human clinical trials based on these measures would be unfeasibly large and prohibitively expensive. In fact, recent data suggest that no clinical trial in HD has ever been sufficiently powered [9]. Neuroimaging biomarkers are obvious candidates as additional outcome measures in future large-scale clinical trials because of their clear relevance to the neuropathology of disease and their increased precision and sensitivity compared with some standard functional measures. Imaging biomarkers must however demonstrate relationships with clinical progression.

Many observational studies and reviews have examined neuroimaging measures cross-sectionally in premanifest and early stage $\mathrm{HD}$, comparing different stages of illness to make assumptions about disease progression. Fewer however have acquired serial scans and assessed longitudinal change directly. It is these findings that are most relevant when considering imaging markers as outcome measures for clinical trials of disease-slowing compounds. This review will summarize the main findings from longitudinal imaging studies in HD utilizing structural MRI, fMRI, PET and DTI. The sensitivity, reproducibility and feasibility of each measure will be discussed.

\section{Search terms}

PubMed and Web of Science databases (October 2011-May 2012) were searched for English language articles containing the terms: Huntington*, imaging, longitudinal, follow-up and/or serial in various combinations. Animal studies were excluded. 24 structural MRI, 3 DTI, 2 fMRI and 13 PET studies were found.

\section{STRUCTURAL MRI}

Structural MRI allows assessment of the macrostructural effects of the underlying neuropathology of HD, namely brain atrophy. Longitudinal MRI studies typically report atrophy rates either in absolute terms (ml per year) or proportional terms (percentage of baseline volume per year). Manual delineation of brain structures, often aided by intensity constraints, is widely accepted to be as close as possible to a 'gold standard' measure, although for practical reasons many groups have moved to using automated methods. Alternatively, mass univariate analyses statistically compare voxel-wise differences across the whole-brain, with no a priori assumptions regarding regions of interest e.g. voxel-based morphometry (VBM) [10]. The simplest method for quantifying change involves subtraction of a follow-up volume from the baseline volume. This however requires analysis at multiple time points which may increase noise, errors and labour demands and so is not ideal for large multi-site longitudinal studies. Automated alternatives include the Boundary Shift Integral (BSI), which calculates volume change at brain-CSF (cerebrospinal fluid) boundaries $[11,12]$ and SIENA software (from the FSL toolbox http://fmrib.ox.ac.uk/fsl [13, 14]) which takes perpendicular profiles at each edge point and compares the derivatives to quantify edge motion and compute percentage brain volume change (PBVC). Quantitative anatomical regional change (Quarc [15, 16]) and voxel-compression maps (VCMs) [17] utilise non-linear registration based on linear elasticity [18] and a compressible viscous fluid model respectively, to produce voxel-wise change maps between serial scans which can also be used to quantify change.

\section{Basal ganglia}

Since the most striking pathological changes in HD are found in the basal ganglia [19], many MRI studies have focused on this region (Table 1). Applying manual delineation, Aylward et al. were the first to demonstrate longitudinal atrophy of this structure in manifest [20] and pre-manifest [21] HD. These findings have been replicated in multiple studies, applying manual, semi- and fully-automated measurement techniques, with both single- and multi-site study designs. Over a 24-month period TRACK-HD found there to be significantly greater atrophy within the caudate of progressors (presymptomatic mutation carriers with an increase in total motor score (TMS) of 5 points 
E.M. Rees et al. / Longitudinal Imaging in HD

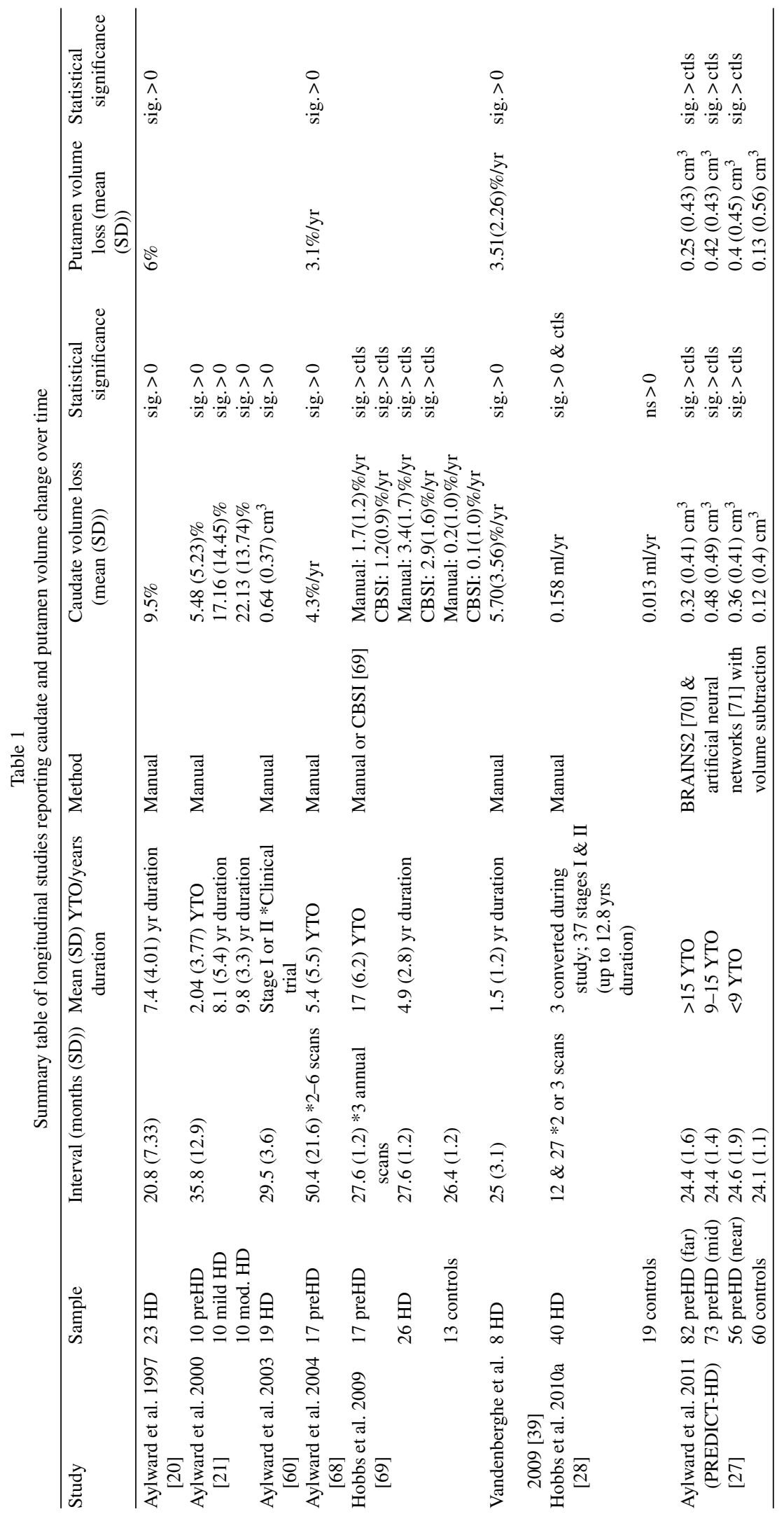




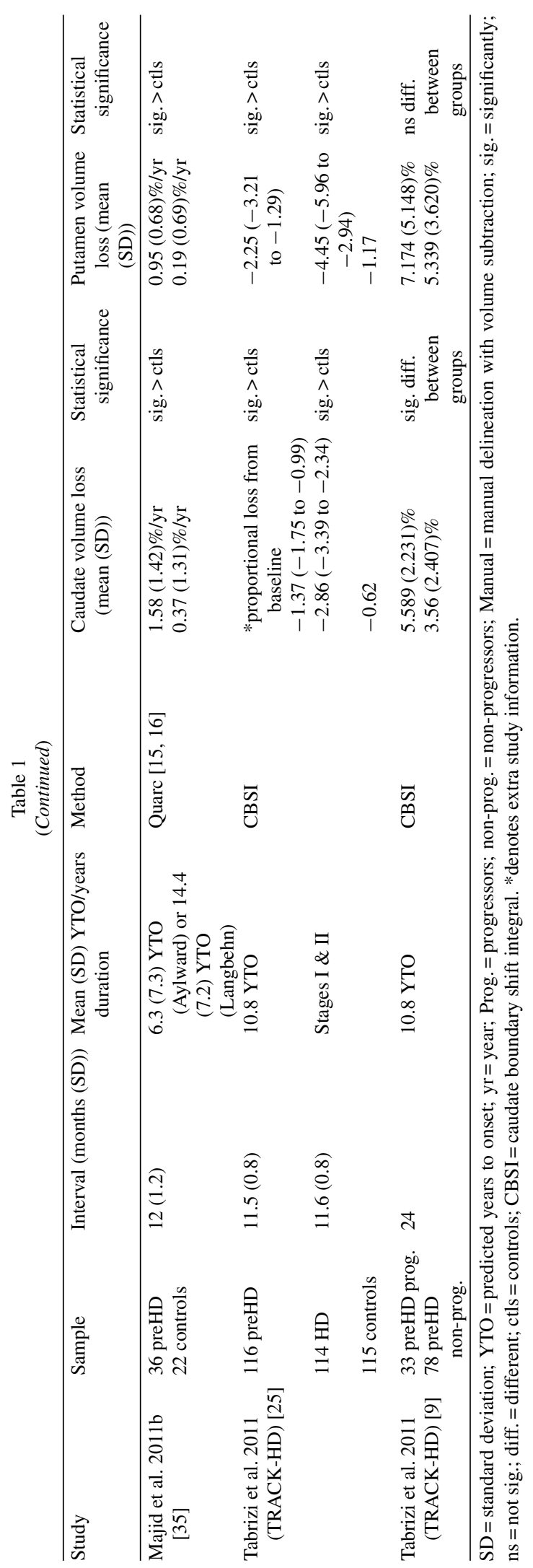


or more, any total functional capacity (TFC) decline, or a new diagnostic confidence score of 4) than nonprogressors [9]. There was no significant difference between groups in putamen atrophy rate. This suggests that caudate atrophy is associated, directly or indirectly, with one or all of these clinical measures. In practical terms, the caudate is attractive as a biomarker as its boundaries are generally well-defined by the intensities in T1 scans, compared with the putamen, and segmentation is therefore facilitated.

\section{Whole-brain}

Table 2 summarizes longitudinal MRI studies reporting whole brain volume. In manifest $\mathrm{HD}$, significantly increased whole-brain atrophy rates have been consistently detected relative to controls [22-25]. Findings are less consistent in preHD with several studies failing to detect significant increases in rates relative to controls $[23,24]$. Those that did were larger cohorts [9, 25-27]. Lack of consistency here is likely to be a result of differences between studies with respect to sample size and the characteristics of the cohorts studied (especially years to onset (YTO)). Over 24 months TRACK-HD found progressors to have significantly higher levels of whole-brain volume loss than nonprogressors, suggesting there is an association with clinical progression [9].

\section{Ventricles}

Ventricular measures are complementary to wholebrain measures, since both reflect the global effects of neurodegeneration. Ventricular expansion of $1.44 \mathrm{ml}$ in the year immediately before disease onset and $1.57 \mathrm{ml}$ in the year immediately after actual disease onset has been reported [28]. These findings are supported by a larger study showing ventricular expansion $0.42 \mathrm{ml}$ greater in preHD than controls and $1.63 \mathrm{ml}$ greater in HD than controls over 12-months [25]. In the TRACKHD study, there was a significant difference in the rate of expansion between preHD progressors and preHD non-progressors [9], all of which suggests an increased rate of atrophy and consequent ventricular enlargement with disease progression. Although ventricular markers are appealing as measurement is facilitated by well-defined boundaries, there is large natural variability in ventricular volume between individuals. There is also some suggestion that ventricular measurements may be more affected by non-disease related changes such as dehydration, hydrocephalus and diuretic therapy [29], which could confound some subtle longitudinal changes.

\section{Grey and white matter}

VBM studies have reported significantly elevated atrophy rates in subcortical GM and selective cortical regions in HD [25, 30, 31] and preHD [25, 32]. Two region-of-interest (ROI) studies failed to detect GM atrophy in preHD [27, 33] but another detected atrophy in manifest HD [34]. More recent work suggests that WM changes are also important in HD, with widespread elevated WM atrophy rates reported using VBM in manifest HD compared to zero [30] and to controls $[25,31]$, whilst in preHD WM atrophy rates were significantly elevated compared to controls in a large cohort [25], but a smaller study failed to detect a difference [31]. Studies delineating the WM have detected significant longitudinal volume loss many years before disease onset relative to zero [33, 34] and to control rates [27]. PREDICT-HD found the frontal lobe WM to be disproportionately affected [27]. In fact, when normal age-related atrophy was taken into account, WM atrophy was greater than striatal atrophy in the HD group, implicating it as a strong, but largely unexplored biomarker candidate. In TRACK-HD, the preHD progressors were found to have significantly higher rates of both GM and WM atrophy than non-progressors suggesting an important correlation between neuroimaging changes and clinical decline [9]. Table 3 summarizes these results.

\section{Correlations with clinical measures}

One potential limitation of structural MRI markers as outcome measures is a lack of certainty over how they relate to clinical decline in HD. Many studies have investigated associations between structural imaging and clinical measures: however, the relationship is often complicated by noise in both domains, a lack of sensitivity to change in either or both, and/or a temporal dissociation between the two (e.g. structural degeneration is evident over a decade prior to the onset of overt clinical signs). It has required large multi-site observational studies such as TRACK-HD, to provide sufficient power to address these relationships. TRACK-HD reported a significant association between atrophy rates and TFC at both 12 and 24 months $[9,25]$. Of the studies in this review, two (from the same cohort) reported a significant association between atrophy rate and the UHDRS TMS 


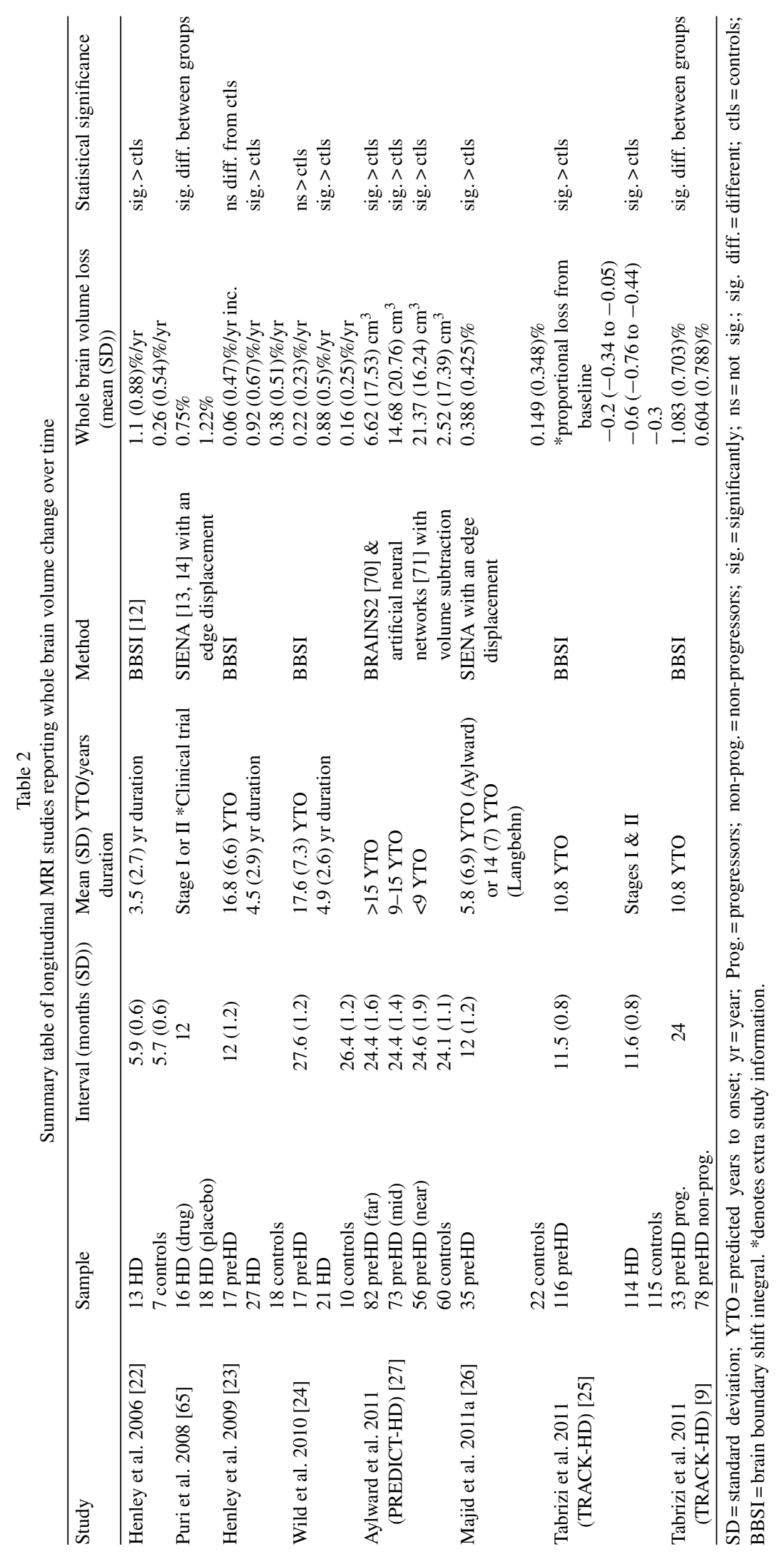




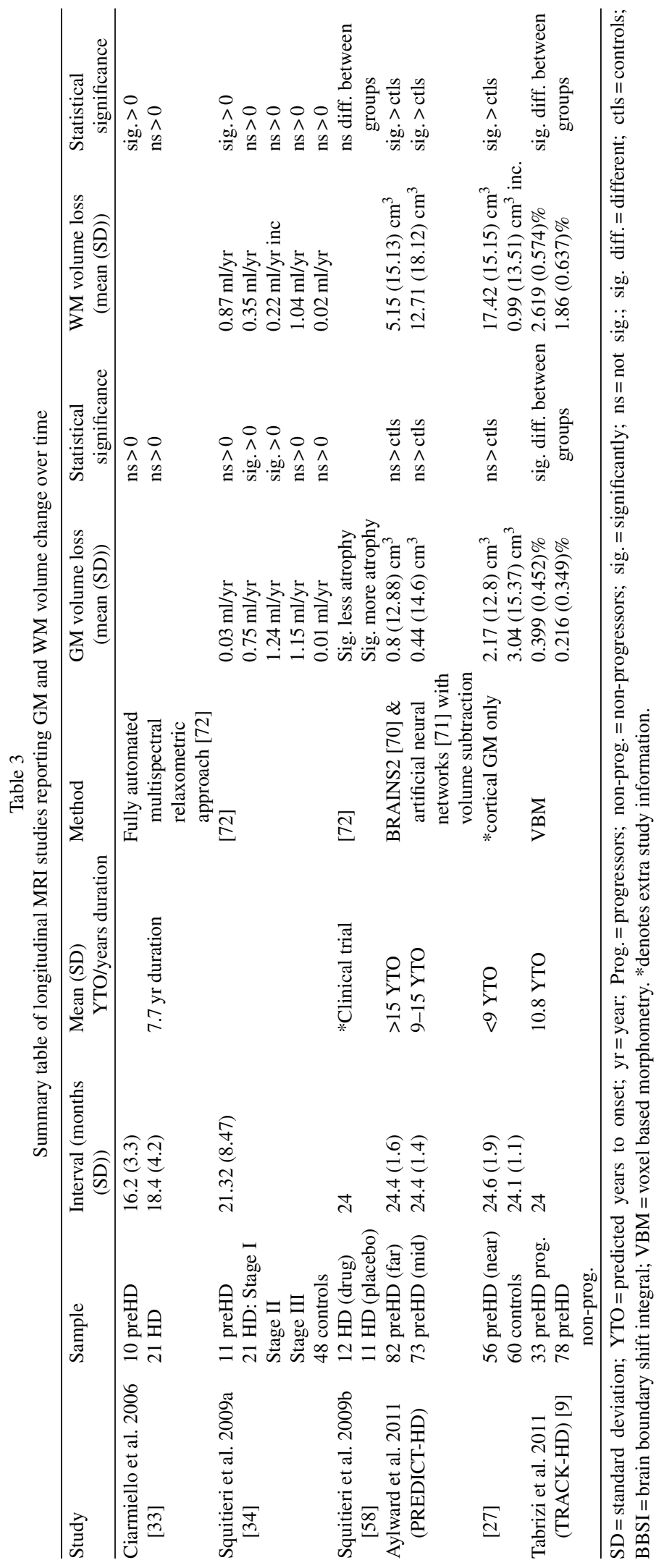


$[9,31]$. Other studies however have failed to replicate this finding [26, 28, 35]. More extensive and focused investigations of the relationship between MRI metrics within clinical and cognitive measures (quantitative motor, oculomotor, cognitive and neuropsychiatric) have been cross-sectional $[36,37]$. These studies have shown strong associations between MRI and disease presentation.

\section{Summary}

Large-scale structural MRI studies have consistently detected global and local atrophy in HD gene-carriers over a decade before symptom onset. Longitudinal imaging of caudate volume is one of the most promising biomarkers for future trials as measurement is reliable and reproducible, and the structure is disproportionately affected by the disease. The TRACK-HD study compared the effect sizes between structural MRI imaging biomarkers and found caudate atrophy to have the highest effect size in both preHD and HD [9]. The putamen is also a strong candidate but rates here are perhaps slightly slower and measurement can be complicated by vessels and poor contrast in this region. PREDICT-HD data suggests that the largest effect sizes in preHD may come from cerebral WM volume change [27] but further studies are required to verify this result. Global measures such as the whole-brain and ventricles show slower rates because they include regions not yet recruited by the disease. These larger regions however have the advantage over local regions of showing the global effects of any intervention.

\section{DIFFUSION MRI}

Diffusion imaging allows assessment of the microstructural effects of the underlying neuropathology of HD. More specifically, diffusion markers are thought to reflect the structural stability of neural tracts within the brain by detecting the extent and coherence of water diffusion, which is largely isotropic through the fibrous WM, more diffuse within the layered GM and fully anisotropic within the viscous CSF. The extent and coherence of water diffusion is altered by the degenerative process.

DTI studies typically report mean diffusivity (MD), fractional anisotropy (FA), axial diffusivity (AD; diffusion parallel to WM fibres) and radial diffusivity (RD; diffusion perpendicular to the primary axis, thought to reflect demyelination). Studies employ either a ROI approach, where the metric is averaged over that par- ticular region, or an automated voxel-wise approach, such as Tract-Based Spatial Statistics (TBSS; http://www/fmrib.ox.ac.uk/fsl/tbss/index.html) where the metric is compared between groups for each voxel within a WM skeleton, containing only the major WM tracts. TBSS is thought to reduce partial volume effects by including only WM voxels which are common to all subjects in the cohort. Despite diffusion metrics often being suggested as biomarkers of disease progression based on cross-sectional findings, only three studies have actually investigated diffusion imaging in a longitudinal setting (Table 4).

Using TBSS significant reductions in FA and AD were detected throughout the brain of HD subjects over one year compared to zero [38]. Changes were particularly notable within subcortical, callosal and frontostriatal tracts. There were also some regions of RD increases, suggesting demyelination. However, this study was small $(n=14)$, used a small number of directions for acquisition, and has not since been replicated. In contrast, despite larger numbers, neither of the two longitudinal ROI DTI studies detected significant changes over one or two years $[39,40]$.

\section{Summary}

Theoretically DTI may offer improved sensitivity compared with macro-structural volumetric analysis, however longitudinal research is at a very early stage in this area. The inconsistency in current findings is most likely due to the methodology used: the metric reported; tissue type studied (GM or WM); varying acquisition parameters (increasing the number of gradient directions increases the quality of the data); TBSS or ROI analyses (ROIs possibly result in more registration errors and partial volume effects); sample size and heterogeneity are also major limitations of these studies. As preliminary work these three studies pose questions that only larger, well-characterised cohorts will be able to address. Longer intervals may also be necessary to pick up changes and multiple scans will help to clarify rate of change.

In general with DTI analyses there are several issues that need to be resolved. Firstly, measures of WM integrity rely on an average value for each voxel and within each voxel there may be multiple fibres travelling in multiple directions (crossing fibres). Imaging techniques with higher angular resolution would improve this. Secondly, the precise nature of the damage underlying MD and FA changes is still unclear, especially with respect to the GM. 
Table 4

A summary table of the longitudinal DTI studies in HD

\begin{tabular}{|c|c|c|c|c|c|c|c|}
\hline Study & $\begin{array}{l}\text { Interval } \\
\quad(\text { months } \\
(\mathrm{SD}))\end{array}$ & Sample & $\begin{array}{c}\text { Mean YTO/years } \\
\text { duration (SD) }\end{array}$ & $\begin{array}{l}\text { Acquisition } \\
\text { parameters }\end{array}$ & Regions studied & $\begin{array}{l}\text { Diffusion } \\
\text { measure }\end{array}$ & $\begin{array}{l}\text { Results (Change } \\
\text { per year) }\end{array}$ \\
\hline \multirow{5}{*}{$\begin{array}{l}\text { Weaver et al. } 2009 \\
\text { [38] }\end{array}$} & 12 & 7 controls & & 32 directions & TBSS skeleton & FA & Controls: $n s>0$ \\
\hline & & 4 preHD & 13.2 YTO & $\begin{array}{l}2 \mathrm{~mm} \text { slice } \\
\text { thickness }\end{array}$ & & & $\begin{array}{l}\text { PreHD \& HD: } \\
\text { decrease - } \\
\text { sig. }>0\end{array}$ \\
\hline & & $3 \mathrm{HD}$ & 2.67 yr duration & & & $\mathrm{AD}$ & $\begin{array}{l}\text { Controls: ns }>0 \\
\text { PreHD \& HD: } \\
\text { decrease - } \\
\text { sig. }>0\end{array}$ \\
\hline & & & & & & $\mathrm{RD}$ & Controls: ns $>0$ \\
\hline & & & & & & & $\begin{array}{l}\text { PreHD \& HD: } \\
\quad \text { increase - ns }>0\end{array}$ \\
\hline \multirow[t]{2}{*}{$\begin{array}{l}\text { Vandenberghe et al. } \\
2009 \text { [39] }\end{array}$} & $25(3.1)$ & $8 \mathrm{HD}$ & $\begin{array}{c}1.5(2.1) \mathrm{yr} \\
\text { duration }\end{array}$ & 3 directions & Caudate & MD & $\begin{array}{l}1.67(3.53) \% / \mathrm{yr} \\
\text { increase - ns }>0\end{array}$ \\
\hline & & & & $\begin{array}{l}4 \mathrm{~mm} \text { slice } \\
\text { thickness }\end{array}$ & Putamen & & $\begin{array}{l}1.91(2.79) \% / \mathrm{yr} \\
\text { increase }-\mathrm{ns}>0\end{array}$ \\
\hline \multirow[t]{4}{*}{$\begin{array}{l}\text { Sritharan et al. } 2010 \\
\text { [40] }\end{array}$} & 12 & $17 \mathrm{HD}$ & $\begin{array}{c}5.4(3.6) \mathrm{yr} \\
\text { duration }\end{array}$ & 28 directions & Corpus callosum & MD & $\mathrm{ns}>0$ or ctls \\
\hline & & 16 controls & & $\begin{array}{l}2.5 \mathrm{~mm} \text { slice } \\
\text { thickness }\end{array}$ & Caudate & & $\mathrm{ns}>0$ or ctls \\
\hline & & & & & Putamen & & $\mathrm{ns}>0$ or ctls \\
\hline & & & & & Thalamus & & $\mathrm{ns}>0$ or ctls \\
\hline
\end{tabular}

$\mathrm{SD}=$ standard deviation; $\mathrm{YTO}=$ estimated years to onset; $\mathrm{yr}=$ year; sig. $>0=$ significantly greater than zero; ctls = controls; $\mathrm{ns}=$ not significantly.

\section{FUNCTIONAL MRI}

Functional imaging uses Blood Oxygenation Level Dependent (BOLD) signal to localise regions of brain activated by a task, discover brain networks that function together to complete certain tasks, and can be used to detect functional abnormalities in individuals with HD compared with controls. There is evidence for slow and subtle cognitive decline in preHD, spreading and becoming more severe with disease progression. Cross-sectional functional studies in preHD have reported lower task-related activations even when performance levels are normal. This is sometimes also accompanied by enhanced cortical activation, often interpreted as neural compensation for dysfunctional circuitry elsewhere [41]. Therefore there is a suggestion that fMRI may be able to pick up early neural dysfunction before morphological changes take place and hence may provide a more sensitive measure than structural or diffusion MRI.

Mass-univariate analysis over the whole-brain can be applied to localise regions of significant activation during a task or differences between groups in restingstate activations. Resting state fMRI is a relatively new and comparatively unexplored method of identifying regional interactions that occur when a subject is not performing an explicit task. Table 5 gives details of the only two longitudinal fMRI studies published in HD to date.

Cross-sectionally, lower activation of the left dorsolateral prefrontal cortex was seen in preHD compared to controls during a working memory task in which gene carriers performed normally [42]. Two years later the same cohort was scanned performing the same task [43]. The authors predicted a further loss in activation caused by disease progression over time; however, there was no evidence of further loss. To date, resting state fMRI has failed to find significant differences between preHD and controls either cross-sectionally or longitudinally [44]. It may be that the paucity of longitudinal fMRI studies in the HD literature is a result of methodological limitations, either in image acquisition, study design or data analysis; the utility of this imaging modality to track disease progression is uncertain but large multi-site studies such as Track On-HD seek to address this issue.

\section{Summary}

No studies have managed to detect significant change over time using fMRI in HD. The lack of longitudinal fMRI studies currently published in the literature may be due to difficulties in ensuring reliability or maximising the signal-to-noise ratio. Test-retest 
Table 5

A summary table of the longitudinal functional MRI studies in HD

\begin{tabular}{|c|c|c|c|c|c|c|}
\hline Study & $\begin{array}{l}\text { Interval (months } \\
\quad(\mathrm{SD}))\end{array}$ & Sample size & $\begin{array}{l}\text { Sample characteristics } \\
(\text { mean YTO }(\mathrm{SD}))\end{array}$ & Task & $\begin{array}{l}\text { Changes in activation } \\
\text { over time }\end{array}$ & Comments \\
\hline \multirow[t]{2}{*}{ Wolf et al. 2011 [43] } & PreHD: 26.2 (2.7) & 13 & $\begin{array}{l}\text { 19.5 YTO (11.2; } \\
\text { Langbehn) }\end{array}$ & Working memory & None found & $\begin{array}{l}\text { Differences } \\
\text { remained } \\
\text { present but } \\
\text { stable }\end{array}$ \\
\hline & Controls: 25 (1.7) & 13 & & & & \\
\hline \multirow[t]{2}{*}{$\begin{array}{l}\text { Seibert et al. } 2012 \\
\text { [44] }\end{array}$} & PreHD: 13.2 (1.2) & 34 & $\begin{array}{l}6.2 \text { YTO }(7.4 ; \text { Aylward }) \\
\text { or } 14.3 \text { YTO }(7.2 ; \\
\text { Langbehn })\end{array}$ & Resting state & None found & $\begin{array}{l}\text { No difference } \\
\text { between groups } \\
\text { cross- } \\
\text { sectionally or } \\
\text { longitudinally }\end{array}$ \\
\hline & Controls: 12 (1.2) & 22 & & & & \\
\hline
\end{tabular}

reliability, multi-site and cross-culture studies are needed to increase confidence in functional change scores. Improvements in scanner stability and technical aspects of functional imaging may be necessary. It might also be that cognitive dysfunction in HD does not evolve uniformly making this unfeasible as a biomarker.

The relationship between the BOLD signal and underlying neuronal activity still remains unclear [45]: both excitatory and inhibitory activation leads to increased metabolism and hence increased signal; much of the metabolic activity is caused by astrocytes, not neurons; and the relationship between the BOLD signal and neural activity varies by site and circuit. This can lead to seemingly contradictory results. A better understanding of this relationship will help to clarify the interpretation of results. Brown \& Eyler [46] discuss methodological and conceptual issues in functional imaging which includes disease-related variability: altered structure and function, vascular effects, task performance level and medication. On top of this the choices of statistics and the methods for correcting for false positives are important sources of variation (this applies to all modalities). Although issues remain to be resolved, it is generally agreed that blood flow is a good index of neural activity and fMRI is clearly a useful technique to explore compensatory mechanisms in HD. As a longitudinal biomarker in HD however fMRI has yet to prove itself.

\section{PET}

PET involves the injection of a radio-labelled ligand specifically designed to bind to particular structures/substances within the brain which can be imaged to detect metabolic and neural changes. PET studies in HD typically quantify dopamine receptor binding/availability or measure glucose metabolism. The substrates and radio-tracers used to image them are listed in Table 6. As with DTI, averaging a metric over the defined ROI and mass univariate methods can be applied to PET data. Table 7 details the longitudinal PET studies in HD.

\section{Dopamine receptor binding}

The only observational study to track D1 receptor binding in HD found that the percentage loss per year was significantly greater than that in controls in both the caudate and putamen, in pre- and early HD groups [47]. More observational studies have assessed D2 receptor binding longitudinally in HD. In early HD the rate of binding potential (BP) decline in the striatum was found to be significantly faster than that in controls over an average of 39 months [47] and VBM detected significant changes from baseline over 29 $( \pm 12.8)$ months [48]. In preHD striatal D2 BP loss was significantly faster than that in controls [47] and significant changes were seen on scans taken with 18-36 month intervals [49]. This result was replicated with 18 and 44 month intervals [50]. Van Oostrom et al. [51] however did not detect significantly increased rates in the putamen compared to the control group D2 binding in a close-to-onset preHD group over an average of 28.8 months.

\section{Glucose metabolism}

Two studies of preHD separately reported reductions in glucose metabolism of $3.1 \% / \mathrm{yr}$ in the caudate (significant compared to control rate [52]) and 2.3\%/yr in the striatum (not significantly greater than zero [49]). A later study found the change in preHD to be significant, this time in the right frontal lobe, caudate and left putamen over 16 months compared to a rate of zero [33]. This same study found the changes to be signif- 
Table 6

The main forms of PET imaging in HD

\begin{tabular}{lllll}
\hline Measurement & Substrate & Radiotracer & Abbreviation & Reported \\
\hline Dopamine receptor density & D2 receptors & 11C-raclopride & 11C/RACLO & Binding potential (BP) \\
& D1 receptors & 11C-SCH & & \\
Glucose metabolism & Glucose & 2-Fluoro-2-deoxyglucose & 18F/FDG-PET & Cerebral metabolic rate of glucose (CMRglu) \\
\hline
\end{tabular}

icant in manifest HD in the right frontal lobe, caudate and putamen over 18 months compared to a rate of zero.

Several studies conducted FDG and RACLO-PET on the same cohorts [49, 50, 53, 54]. Overall, these results suggest that RACLO-PET is more sensitive to change than FDG-PET but there seems to be a correlation between D2 binding and glucose metabolism suggesting that dysfunction in glucose metabolism maybe related to $\mathrm{D} 2$ receptor loss.

\section{HD-related metabolic covariance pattern (HDRP)}

A covariance pattern is an imaging pattern that is characteristic of a specific group e.g. HD. The HDRP is dominated by striatal hypo-metabolism and hypermetabolism in the cerebellum, thalamus, occipital and parietal cortices and can be used to distinguish preHD and HD from control brains. It is thought that this represents compensatory processes in functional brain circuits. One research group has looked at the evolution of this pattern over time and found slightly different HDRPs at each time point studied $[50,55,56]$. There was found to be a significant increase in HDRP expression over 18 months, relative to baseline, but a decrease over 44 months (still above baseline). Although unstable, these patterns may help us to understand the development of deteriorating metabolic functioning before disease onset, but more work is required to fully characterise these patterns.

\section{Correlations with clinical measures}

For PET to be a relevant biomarker the measures must correlate with clinical function. From the FDGPET studies in this review one reports a significant correlation between glucose uptake and a large battery of neuropsychological, motor and psychiatric tests [57] and a second shows a linear correlation with the severity of motor and behavioural scores [58]. Two report clinical correlations with dopamine receptor binding: change in UHDRS motor scores correlated significantly with changes in D1 receptor binding in the caudate nucleus and putamen [53]; and results from a test of executive function correlated significantly with striatal and putamen D2 binding [48]. These results suggest that PET markers are associated with clinical measures.

\section{Summary}

Within the striatum in preHD, there is some evidence that $\mathrm{D} 2$ receptor loss occurs at a faster rate than the accumulation of metabolic dysfunction, suggesting that disturbance of glucose metabolism is secondary to dysfunction and loss of the medium spiny neurons. Both metabolic and dopamine receptor decline appears to spread from the striatum out towards the cortex with disease progression. The PET studies in this review are limited most prominently by sample size, which may be a result of the intrusive injection of radioligands necessary for this imaging technique, availability of equipment, stability of ligands and/or high costs. These factors would also limit the use of this technique as an outcome measure in large-scale trials.

It is known that FDG-PET reflects glial as well as neuronal activity and is influenced by cortical inputs and inflammatory processes, hence making it less specific than PET imaging of dopamine receptors. Another issue in HD is atrophy correction. Hypometabolism and atrophy often co-occur (i.e. less brain volume is likely to equate to fewer binding regions simply due to the amount of brain tissue) but hypometabolism can also appear without atrophic changes. By correcting for atrophy it is possible to differentiate authentic hypometabolism (decrease of glucose consumption per unit volume of GM) from that due to atrophy [59]. Atrophy correction is achieved by co-registering the structural data to correct for volume loss. One preliminary study found striatal hypometabolism to disappear to a large extent after atrophy correction [56].

\section{CLINICAL TRIALS}

\section{Neural grafts}

Two cohorts have undergone striatal grafting surgery with pre- and post-operative PET scans. The first was comprised of five patients, three of whom showed 


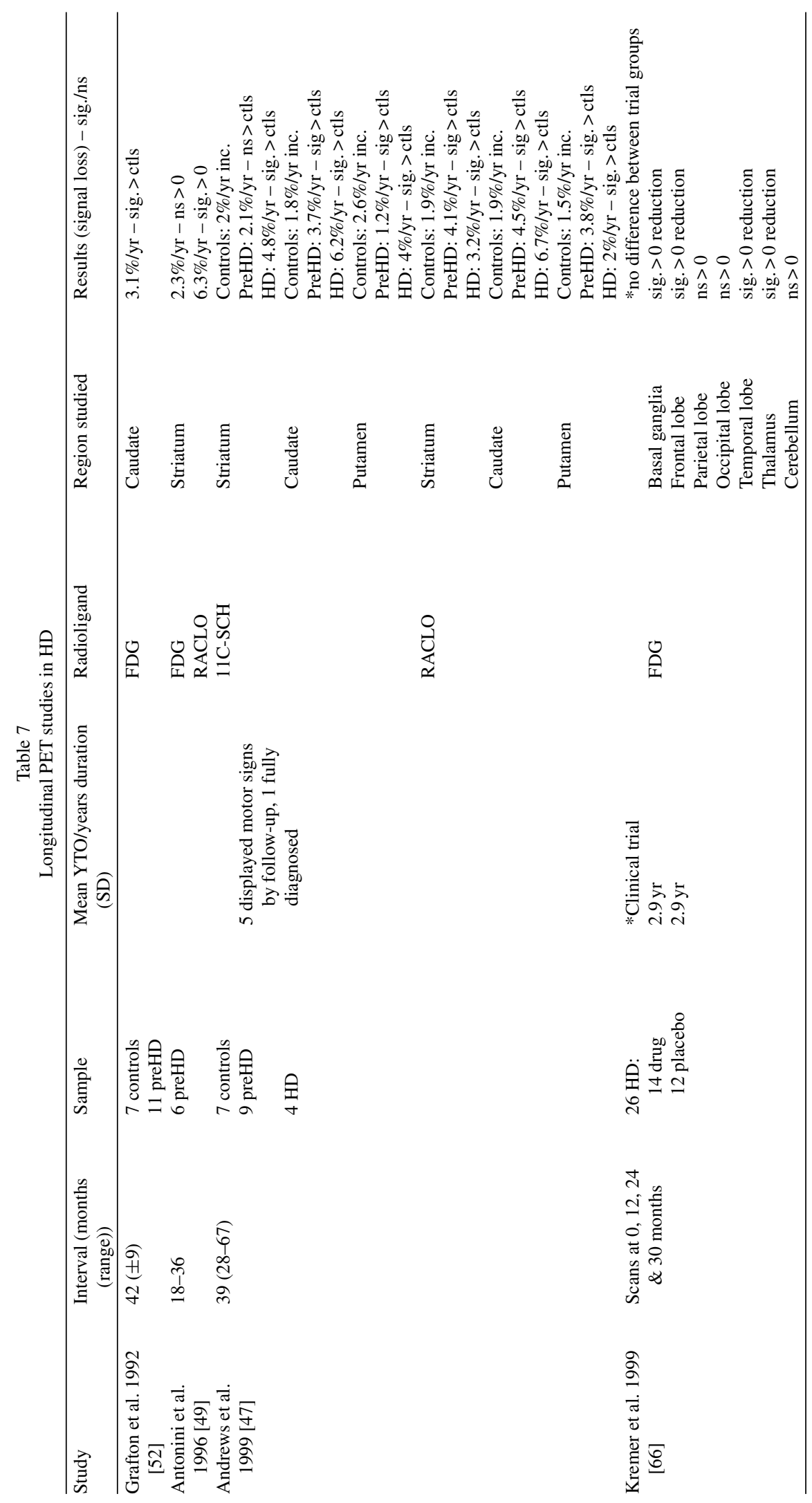




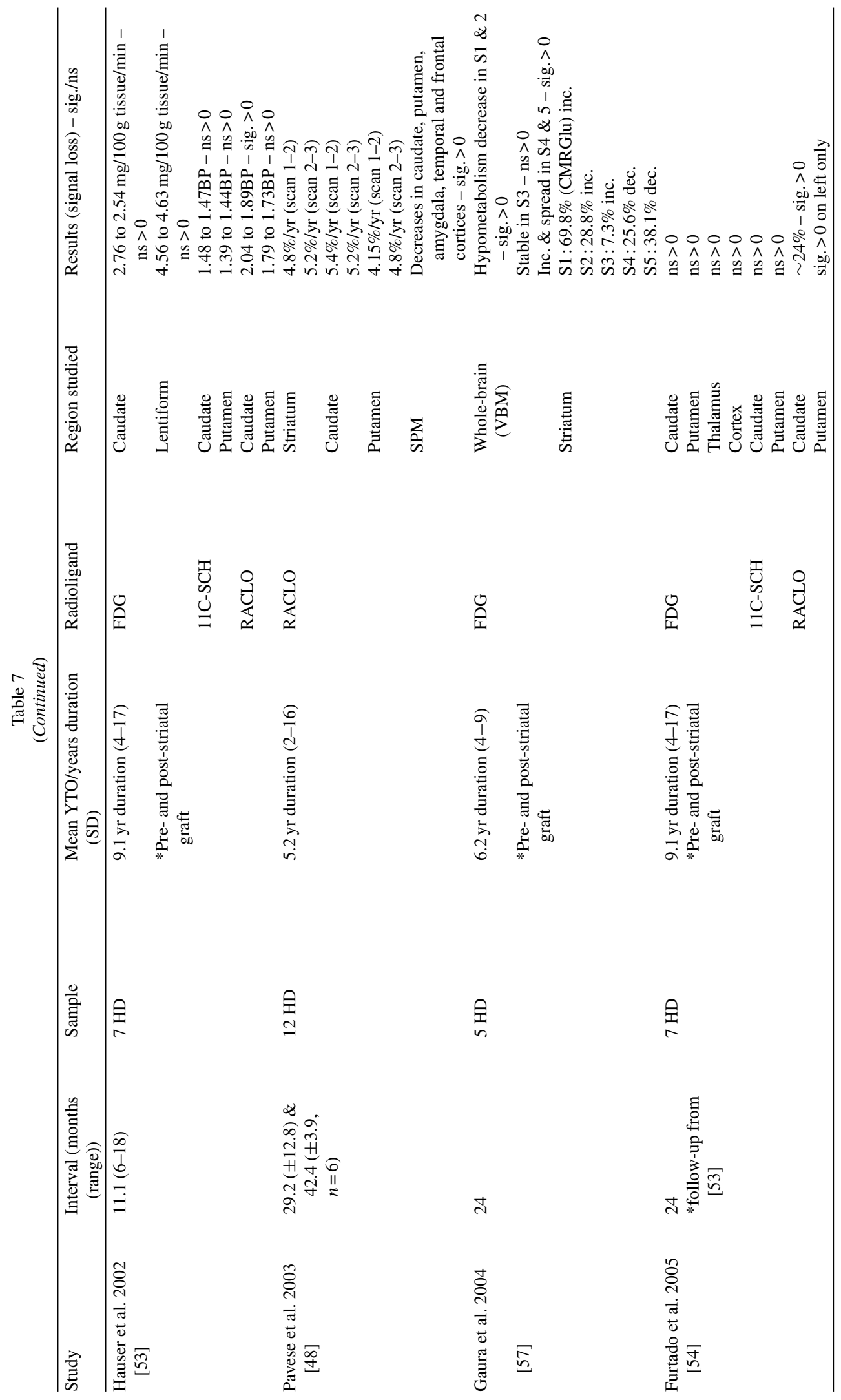




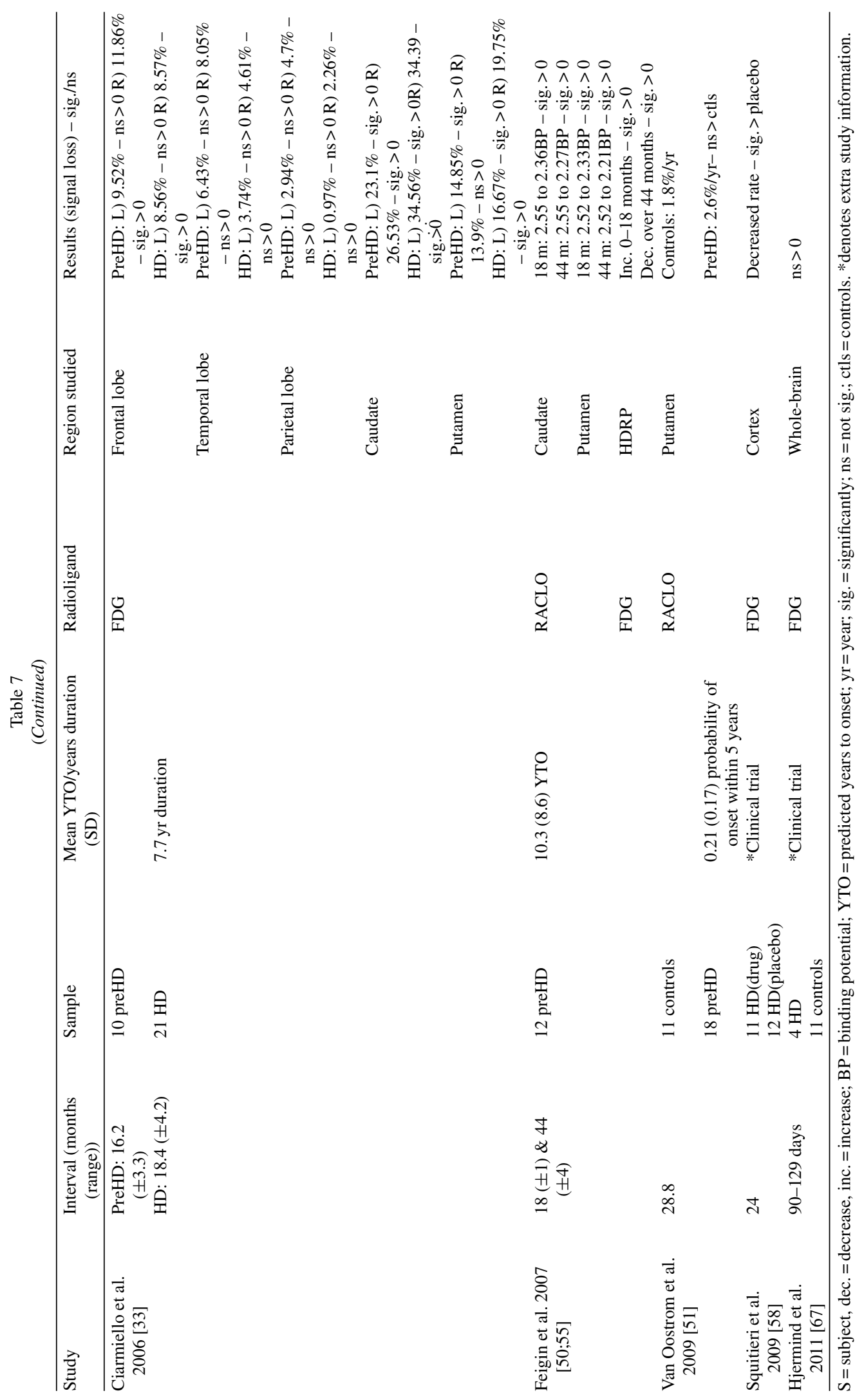


reduction of striatal and cortical hypometabolism and two of whom showed progressing hypometabolism [57] over the two-year follow-up. Clinical changes in this study were associated with changes in brain metabolism. In the second cohort of seven HD patients, D1 and D2 receptor binding were used to assess the effects of striatal grafts at 11 [53] and 24 months [54]. D1 BP change was not significant over 11 months (pre- and post-striatal graft) in the caudate or putamen. Twelve months later, still no significant change was detected from baseline. There was significant loss of D2 BP in the caudate pre- and post-graft but this was not significant in the putamen. At 44 months postgraft there was significant loss in the caudate and left putamen only. These results could potentially reflect a beneficial effect of the grafts, stabilising D1 receptor loss. However, more longitudinal observational studies are needed to establish baseline rates before robust conclusions can be drawn. Additionally, the imaging measures were not correlated with clinical metrics therefore it is unclear what relation these changes have to disease progression.

These studies demonstrate the feasibility of transplanted cells to reconstruct damaged circuitry in HD and the ability of PET imaging to track the survival and possible beneficial effects of grafts. There were, however, multiple adverse events associated with this treatment, including a higher risk for subdural haemorrhages.

\section{Pilot clinical trials}

TRACK-HD and PREDICT-HD are large, multisite, observational studies utilising longitudinal MR imaging which have been designed to imitate clinical trial set-ups. The challenges associated with largescale organisation, quality control and assurance, data management, anonymization, storage, analysis and data dissemination have been addressed by these studies. Inter-scanner differences and consistency of acquisition protocol have been highlighted as important areas to consider. Both studies have also provided sample size recommendations for future trials, dependent on the effect size required for hypothetical treatments ranging from $20-100 \%$ efficacy for a 2 year, 2 group trial $[9,27]$.

At present the clinical trials in the literature applying MRI and/or PET as outcome measures have not come near to the size or level of complexity of these studies and were not designed to detect beneficial therapeutic effects or efficacy. There are currently only five published pilot trials:
The Riluzole trial $(n=23)$ [58] applied both MRI and FDG-PET measures as end-points. Riluzole was found to improve clinical scores, protect from glucose hypometabolism, reduce GM volume loss and increase production of neurotrophins compared with the placebo group over 2 years. This was followed-up by a large $(n=537)$, 3-year randomized control trial (using motor and TFC scores as endpoints but no imaging). No neuroprotective or beneficial symptomatic effects were demonstrated.

The pilot trial of Co-enzyme Q10 with Remacemide $(n=19)$ [60] established that caudate volume (from MRI data) is a feasible end-point and a power analysis indicated that, to demonstrate treatment effectiveness in slowing caudate atrophy by $50 \%$, relatively small numbers of subjects would be needed in clinical trials with this as an outcome measure. If treatment was expected to be less effective, larger sample sizes would be needed. A randomised trial $(n=347)$ using TFC as the primary measure of efficacy (no imaging) reported no slowing in functional decline over 30 months [61].

The ethyl-EPA pilot study $(n=4)$ [62] used registered difference MRI images, composed of the follow-up scan minus the baseline. Over 6-months, the placebo was associated with progressive cerebral atrophy (evident around the ventricles) whereas the drug group showed the reverse process. Two phase III trials ( $n=121$ and $n=316)$ found negative results with TMS as the efficacy scale [63, 64]. A subgroup of the HD patients who took part in the first largescale trial underwent baseline, 6-month and 12-month follow-up MRI scans. Analysis of these images [65] suggested a reduction in global cerebral atrophy in the drug group compared with the placebo group over the first 6-months but a similar rate in both groups in the second 6-month period. Local analyses revealed differences between group atrophy rates in the head of the caudate and the posterior thalamus.

The Lamotrigine $(n=26)$ [66] pilot study utilised FDG-PET. Both placebo and drug groups showed significantly increased hypometabolism in the basal ganglia, frontal and temporal lobes and thalamus compared to baseline between serial scans up to 30 months apart, yet no treatment effect was detected (assessed by TFC, QNE and FDG-PET). To our knowledge no phase III trial of Lamotrigine has been conducted.

The Memantine trial $(n=4)$ [67] also used FDGPET. Four HD patients showed no neuropsychological or metabolic changes during 3-4 months treatment. One patient prolonged treatment for 18 months and 
showed no deterioration, whilst those that stopped treatment after three to four months had minor progression on all cognitive domains tested, suggesting there may be a slight benefit. A large phase III trial has been completed with assessments of cognitive and motor symptoms as end-points, but the full results have not yet been made public.

In summary, to date there have been no large-scale trials using both imaging and clinical measures as outcomes, with clinical measures such as TFC remaining the preferred metric. To our knowledge no therapeutic in a phase III trial has demonstrated a statistically significant attenuation of disease progression.

\section{CONCLUSION}

\section{Study set-up}

A major point that has emerged from the literature on longitudinal imaging is the need for a set-up phase for multi-site studies and clinical trials. There are several issues that need to be addressed: (1) the study design must consider sample size and characterisation (it has been suggested that all clinical trials in HD to date have been underpowered [9]); (2) motion artefacts, a particular problem in manifest HD, need to be minimised by consideration of disease progression during the course of the study, as well as employing a quality assurance procedure during scan acquisition and applying a thorough quality control procedure to all acquired scans, with rescans obtained where necessary; (3) controlling for inter-site scanner differences by optimising image analysis procedures for the study data and ensuring consistency in scan acquisition. Well-designed scanning parameters and optimised data processing steps, appropriate for the aims of the study, are essential to ensure good quality data. It is currently unclear whether field strength significantly affects longitudinal findings but this should also be a consideration. Fullyautomated analysis methods are preferable for large samples for practical reasons; however, it is important that such techniques are fully validated for use in atrophied HD brains, generally by detailed head-to-head comparisons with established, manual imaging methods, to ensure confidence in findings and reduce the possibility of spurious results.

\section{Discussion}

To conclude we must return to the characteristics of an ideal biomarker: reliable, reproducible, minimally invasive, widely available, low variability in the normal population, linear change with disease progression and predictable response to an intervention which modifies the disease. The imaging modalities that currently best fit this description are structural MRI and PET. Within each modality, caudate volume and RACLOPET could be argued to be the best options at present. PET is more expensive, less widely available and may not be as sensitive to longitudinal change but has the advantage of being able to target specific molecules. Longitudinal DTI and fMRI require further exploration in large multi-site observational studies before their use in a clinical trial environment could be considered. More information is required to optimise these modalities in terms of repeatability, reliability and longitudinal signal-to-noise however both show potential in terms of scientific interest and as biomarkers. Feasibility of imaging measures for use in future clinical trials must also be considered. All imaging modalities are logistically complicated and expensive compared with standard clinical or cognitive end-points and their sensitivity is generally reduced in individuals with later stage HD due to movement. Nevertheless, imaging has several advantages including the ability to track progression in the pre-manifest stage before any detectable clinical or cognitive change. Strong support for the use of structural MRI comes from the TRACK-HD study, which compared imaging, cognitive, quantitative motor and neuropsychiatric measures and found imaging variables to have the largest effect sizes [9].

Overall, neuroimaging shows real promise for characterising the progression of HD and may be qualified to assess drug efficacy in clinical trials of putative disease-slowing compounds in the future. As imaging metrics are an indirect measure of neuronal activity/health which cannot be assumed to relate directly to clinical progression, other measures will be needed alongside them e.g. TFC and/or quality-of-life scales, particularly in clinical trials of early manifest individuals who are likely to be the most suitable population for many of these trials. The advantages and disadvantages of the inclusion of imaging in clinical trials must be seriously considered, with study design and choice of imaging modality based on the theoretical effect of the drug being tested.

\section{REFERENCES}

[1] Rawlins M. Huntington's disease out of the closet? Lancet. 2010;376(9750):1372-3.

[2] Unified Huntington's Disease Rating Scale: Reliability and consistency. Huntington Study Group. Mov Disord. 1996;11(2):136-42. 
[3] Ross CA, Tabrizi SJ. Huntington's disease: From molecular pathogenesis to clinical treatment. Lancet Neurol. 2011;10(1):83-98.

[4] Atkinson AJ, Colburn WA, DeGruttola VG, Demets DL, Downing GJ, Hoth DF. et al. Biomarkers and surrogate endpoints: Preferred definitions and conceptual framework. Clinical Pharmacology \& Therapeutics. 2001;69(3): 89-95.

[5] Folstein SE, Jensen B, Leigh RJ, Folstein MF. The measurement of abnormal movement: Methods developed for Huntington's disease. Neurobehav Toxicol Teratol. 1983;5(6):605-9.

[6] Brandt J, Bylsma FW, Gross R, Stine OC, Ranen N, Ross CA. Trinucleotide repeat length and clinical progression in Huntington's disease. Neurology. 1996;46(2):527-31.

[7] Giordani B, Berent S, Boivin MJ, Penney JB, Lehtinen S, Markel DS. et al. Longitudinal neuropsychological and genetic linkage analysis of persons at risk for Huntington's disease. Arch Neurol. 1995;52(1):59-64.

[8] Craufurd D, Snowden J. Neuropsychological and neuropsychiatric aspects of Huntington's Disease. Oxford, Oxford University Press; 2002.

[9] Tabrizi SJ, Reilmann R, Roos RA, Durr A, Leavitt B, Owen G. et al. Potential endpoints for clinical trials in premanifest and early Huntington's disease in the TRACK-HD study: Analysis of 24 month observational data. Lancet Neurol. 2011.

[10] Ashburner J, Friston KJ. Voxel-based morphometry - The methods. Neuroimage. 2000;11(6):805-21.

[11] Fox NC, Freeborough PA. Brain atrophy progression measured from registered serial MRI: Validation and application to Alzheimer's disease. J Magn Reson Imaging. 1997;7(6):1069-75.

[12] Freeborough PA, Fox NC. The boundary shift integral: An accurate and robust measure of cerebral volume changes from registered repeat MRI. IEEE Trans Med Imaging. 1997;16(5):623-9.

[13] Smith SM, De SN, Jenkinson M, Matthews PM. Normalized accurate measurement of longitudinal brain change. J Comput Assist Tomogr. 2001;25(3):466-75.

[14] Smith SM, Zhang Y, Jenkinson M, Chen J, Matthews PM, Federico A. et al. Accurate, robust, and automated longitudinal and cross-sectional brain change analysis. Neuroimage. 2002; 17(1):479-89.

[15] Holland D, Brewer JB, Hagler DJ, Fennema-Notestine C, Dale AM. Subregional neuroanatomical change as a biomarker for Alzheimer's disease. Proc Natl Acad Sci U S A. 2009;106(49):20954-9.

[16] Holland D, Dale AM. Nonlinear registration of longitudinal images and measurement of change in regions of interest. Med Image Anal. 2011;15(4):489-97.

[17] Freeborough PA, Fox NC. Modeling brain deformations in Alzheimer disease by fluid registration of serial 3D MR images. J Comput Assist Tomogr. 1998;22(5):838-43.

[18] Holland D, Hagler DJ, Fennema-Notestine C, Dale AM. Longitudinal nonlinear registration and quantitative analysis of change in whole brain and regions of interest. Alzheimer's \& Dementia: The Journal of the Alzheimer's Association 4[4]. 2008.

[19] Halliday GM, McRitchie DA, Macdonald V, Double KL, Trent RJ, McCusker E. Regional specificity of brain atrophy in Huntington's disease. Exp Neurol. 1998;154(2): 663-72.

[20] Aylward EH, Li Q, Stine OC, Ranen N, Sherr M, Barta PE. et al. Longitudinal change in basal ganglia volume in patients with Huntington's disease. Neurology. 1997;48(2):394-9.
[21] Aylward EH, Codori AM, Rosenblatt A, Sherr M, Brandt J, Stine OC. et al. Rate of caudate atrophy in presymptomatic and symptomatic stages of Huntington's disease. Mov Disord. 2000;15(3):552-60.

[22] Henley SM, Frost C, MacManus DG, Warner TT, Fox NC, Tabrizi SJ. Increased rate of whole-brain atrophy over 6 months in early Huntington disease. Neurology. 2006;67(4):694-6.

[23] Henley SM, Wild EJ, Hobbs NZ, Frost C, MacManus DG, Barker RA. et al. Whole-brain atrophy as a measure of progression in premanifest and early Huntington's disease. Mov Disord. 2009;24(6):932-6.

[24] Wild EJ, Henley SM, Hobbs NZ, Frost C, MacManus DG, Barker RA. et al. Rate and acceleration of whole-brain atrophy in premanifest and early Huntington's disease. Mov Disord. 2010;25(7):888-95.

[25] Tabrizi SJ, Scahill RI, Durr A, Roos RA, Leavitt BR, Jones R. et al. Biological and clinical changes in premanifest and early stage Huntington's disease in the TRACK-HD study: The 12month longitudinal analysis. Lancet Neurol. 2011;10(1):3142.

[26] Majid DS, Stoffers D, Sheldon S, Hamza S, Thompson WK, Goldstein J. et al. Automated structural imaging analysis detects premanifest Huntington's disease neurodegeneration within 1 year. Mov Disord. 2011;26(8):1481-8.

[27] Aylward EH, Nopoulos PC, Ross CA, Langbehn DR, Pierson RK, Mills JA. et al. Longitudinal change in regional brain volumes in prodromal Huntington disease. J Neurol Neurosurg Psychiatry. 2011;82(4):405-10.

[28] Hobbs NZ, Barnes J, Frost C, Henley SM, Wild EJ, Macdonald $\mathrm{K}$. et al. Onset and progression of pathologic atrophy in Huntington disease: A longitudinal MR imaging study. AJNR Am J Neuroradiol. 2010;31(6):1036-41.

[29] Schott JM, Price SL, Frost C, Whitwell JL, Rossor MN, Fox NC. Measuring atrophy in Alzheimer disease: A serial MRI study over 6 and 12 months. Neurology. 2005;65(1):119-24.

[30] Ruocco HH, Bonilha L, Li LM, Lopes-Cendes I, Cendes F. Longitudinal analysis of regional grey matter loss in Huntington disease: Effects of the length of the expanded CAG repeat. J Neurol Neurosurg Psychiatry. 2008;79(2):130-5.

[31] Hobbs NZ, Henley SM, Ridgway GR, Wild EJ, Barker RA, Scahill RI. et al. The progression of regional atrophy in premanifest and early Huntington's disease: A longitudinal voxel-based morphometry study. J Neurol Neurosurg Psychiatry. 2010;81(7):756-63.

[32] Kipps CM, Duggins AJ, Mahant N, Gomes L, Ashburner J, McCusker EA. Progression of structural neuropathology in preclinical Huntington's disease: A tensor based morphometry study. J Neurol Neurosurg Psychiatry. 2005;76(5):650-5.

[33] Ciarmiello A, Cannella M, Lastoria S, Simonelli M, Frati L, Rubinsztein DC. et al. Brain white-matter volume loss and glucose hypometabolism precede the clinical symptoms of Huntington's disease. J Nucl Med. 2006;47(2):215-22.

[34] Squitieri F, Cannella M, Simonelli M, Sassone J, Martino T, Venditti E. et al. Distinct brain volume changes correlating with clinical stage, disease progression rate, mutation size, and age at onset prediction as early biomarkers of brain atrophy in Huntington's disease. CNS Neurosci Ther. 2009;15(1):1-11.

[35] Majid DS, Aron AR, Thompson W, Sheldon S, Hamza S, Stoffers D. et al. Basal ganglia atrophy in prodromal Huntington's disease is detectable over one year using automated segmentation. Mov Disord. 2011;26(14):2544-51

[36] Scahill RI, Hobbs NZ, Say MJ, Bechtel N, Henley SM, Hyare H. et al. Clinical impairment in premanifest and early 
Huntington's disease is associated with regionally specific atrophy. Hum Brain Mapp. 2011. DOI: 10.1002/hbm.21449

[37] Bechtel N, Scahill RI, Rosas HD, Acharya T, van den Bogaard SJ, Jauffret C. et al. Tapping linked to function and structure in premanifest and symptomatic Huntington disease. Neurology. 2010;75(24):2150-60.

[38] Weaver KE, Richards TL, Liang O, Laurino MY, Samii A, Aylward EH. Longitudinal diffusion tensor imaging in Huntington's Disease. Exp Neurol. 2009;216(2):525-9.

[39] Vandenberghe W, Demaerel P, Dom R, Maes F. Diffusionweighted versus volumetric imaging of the striatum in early symptomatic Huntington disease. J Neurol. 2009;256(1): 109-14.

[40] Sritharan A, Egan GF, Johnston L, Horne M, Bradshaw JL, Bohanna I. et al. A longitudinal diffusion tensor imaging study in symptomatic Huntington's disease. J Neurol Neurosurg Psychiatry. 2010;81(3):257-62.

[41] Paulsen JS. Functional imaging in Huntington's disease. Exp Neurol. 2009;216(2):272-7.

[42] Wolf RC, Vasic N, Schonfeldt-Lecuona C, Landwehrmeyer GB, Ecker D. Dorsolateral prefrontal cortex dysfunction in presymptomatic Huntington's disease: Evidence from eventrelated fMRI. Brain. 2007;130(Pt 11):2845-57.

[43] Wolf RC, Sambataro F, Vasic N, Wolf ND, Thomann PA, Landwehrmeyer GB. et al. Longitudinal functional magnetic resonance imaging of cognition in preclinical Huntington's disease. Exp Neurol. 2011;231(2):214-22.

[44] Seibert TM, Majid DS, Aron AR, Corey-Bloom J, Brewer JB. Stability of resting fMRI interregional correlations analyzed in subject-native space: A one-year longitudinal study in healthy adults and premanifest Huntington's disease. Neuroimage. 2011;59(3):2452-63.

[45] Tagamets MA, Horwitz B. Interpreting PET and fMRI measures of functional neural activity: The effects of synaptic inhibition on cortical activation in human imaging studies. Brain Res Bull. 2001;54(3):267-73.

[46] Brown GG, Eyler LT. Methodological and conceptual issues in functional magnetic resonance imaging: Applications to schizophrenia research. Annu Rev Clin Psychol. 2006;2: 51-81.

[47] Andrews TC, Weeks RA, Turjanski N, Gunn RN, Watkins LH, Sahakian B. et al. Huntington's disease progression. PET and clinical observations. Brain. 1999;122(Pt 12):2353-63.

[48] Pavese N, Andrews TC, Brooks DJ, Ho AK, Rosser AE, Barker RA. et al. Progressive striatal and cortical dopamine receptor dysfunction in Huntington's disease: A PET study. Brain. 2003;126(Pt 5):1127-35.

[49] Antonini A, Leenders KL, Spiegel R, Meier D, Vontobel P, Weigell-Weber M. et al. Striatal glucose metabolism and dopamine D2 receptor binding in asymptomatic gene carriers and patients with Huntington's disease. Brain. 1996;119 (Pt 6):2085-95.

[50] Feigin A, Tang C, Ma Y, Mattis P, Zgaljardic D, Guttman M. et al. Thalamic metabolism and symptom onset in preclinical Huntington's disease. Brain. 2007;130(Pt 11):2858-67.

[51] van Oostrom JC, Dekker M, Willemsen AT, de Jong BM, Roos RA, Leenders KL. Changes in striatal dopamine D2 receptor binding in pre-clinical Huntington's disease. Eur J Neurol. 2009;16(2):226-31.

[52] Grafton ST, Mazziotta JC, Pahl JJ, St George-Hyslop P, Haines JL, Gusella J. et al. Serial changes of cerebral glucose metabolism and caudate size in persons at risk for Huntington's disease. Arch Neurol. 1992;49(11):1161-7.

[53] Hauser RA, Furtado S, Cimino CR, Delgado H, Eichler S, Schwartz S. et al. Bilateral human fetal striatal transplantation in Huntington's disease. Neurology. 2002;58(5): 687-95.

[54] Furtado S, Sossi V, Hauser RA, Samii A, Schulzer M, Murphy CB. et al. Positron emission tomography after fetal transplantation in Huntington's disease. Ann Neurol. 2005;58(2):331-7.

[55] Ma Y, Feigin A, Rachakonda S, Dhawan V, Eidelberg D. Evolution of metabolic brain networks in presymptomatic Huntington's disease: A longitudinal PET study. J Nucl Med. 2006;47:209.

[56] Ma Y, Eidelberg D. Functional imaging of cerebral blood flow and glucose metabolism in Parkinson's disease and Huntington's disease. Mol Imaging Biol. 2007;9(4):223-33.

[57] Gaura V, Bachoud-Levi AC, Ribeiro MJ, Nguyen JP, Frouin $\mathrm{V}$, Baudic S. et al. Striatal neural grafting improves cortical metabolism in Huntington's disease patients. Brain. 2004;127(Pt 1):65-72.

[58] Squitieri F, Orobello S, Cannella M, Martino T, Romanelli P, Giovacchini G. et al. Riluzole protects Huntington disease patients from brain glucose hypometabolism and grey matter volume loss and increases production of neurotrophins. Eur J Nucl Med Mol Imaging. 2009;36(7):1113-20.

[59] Labbe C, Froment JC, Kennedy A, Ashburner J, Cinotti L. Positron emission tomography metabolic data corrected for cortical atrophy using magnetic resonance imaging. Alzheimer Dis Assoc Disord. 1996;10(3):141-70.

[60] Aylward EH, Rosenblatt A, Field K, Yallapragada V, Kieburtz $\mathrm{K}, \mathrm{McDermott} \mathrm{M}$. et al. Caudate volume as an outcome measure in clinical trials for Huntington's disease: A pilot study. Brain Res Bull. 2003;62(2):137-41.

[61] A randomized, placebo-controlled trial of coenzyme Q10 and remacemide in Huntington's disease. Neurology. 2001;57(3):397-404.

[62] Puri BK, Bydder GM, Counsell SJ, Corridan BJ, Richardson AJ, Hajnal JV. et al. MRI and neuropsychological improvement in Huntington disease following ethyl-EPA treatment. Neuroreport. 2002;13(1):123-6.

[63] Puri BK, Leavitt BR, Hayden MR, Ross CA, Rosenblatt A, Greenamyre JT. et al. Ethyl-EPA in Huntington disease: A double-blind, randomized, placebo-controlled trial. Neurology. 2005;65(2):286-92

[64] Randomized controlled trial of ethyl-eicosapentaenoic acid in Huntington disease: The TREND-HD study. Arch Neurol 2008;65(12):1582-9.

[65] Puri BK, Bydder GM, Manku MS, Clarke A, Waldman AD, Beckmann CF. Reduction in cerebral atrophy associated with ethyl-eicosapentaenoic acid treatment in patients with Huntington's disease. J Int Med Res. 2008;36(5):896-905.

[66] Kremer B, Clark CM, Almqvist EW, Raymond LA, Graf P, Jacova C. et al. Influence of lamotrigine on progression of early Huntington disease: A randomized clinical trial. Neurology. 1999;53(5):1000-11.

[67] Hjermind LE, Law I, Jonch A, Stokholm J, Nielsen JE. Huntington's disease: Effect of memantine on FDGPET brain metabolism? J Neuropsychiatry Clin Neurosci. 2011;23(2):206-10.

[68] Aylward EH, Sparks BF, Field KM, Yallapragada V, Shpritz $\mathrm{BD}$, Rosenblatt A. et al. Onset and rate of striatal atrophy in preclinical Huntington disease. Neurology. 2004;63(1):6672.

[69] Hobbs NZ, Henley SM, Wild EJ, Leung KK, Frost C, Barker RA. et al. Automated quantification of caudate atrophy by local registration of serial MRI: Evaluation and application in Huntington's disease. Neuroimage. 2009;47(4): 1659-65. 
[70] Magnotta VA, Harris G, Andreasen NC, O'Leary DS, Yuh WT, Heckel D. Structural MR image processing using the BRAINS2 toolbox. Comput Med Imaging Graph. 2002;26(4):251-64.

[71] Powell S, Magnotta VA, Johnson H, Jammalamadaka VK, Pierson R, Andreasen NC. Registration and machine learning-based automated segmentation of subcortical and cerebellar brain structures. Neuroimage. 2008;39(1):238-47.

[72] Alfano B, Brunetti A, Covelli EM, Quarantelli M, Panico MR, Ciarmiello A. et al. Unsupervised, automated segmentation of the normal brain using a multispectral relaxometric magnetic resonance approach. Magn Reson Med. 1997;37(1):84-93. 